\title{
Targeting cancer stem cells in gastric cancer
}

\author{
This article was published in the following Dove Press journal: \\ Gastrointestinal Cancer: Targets and Therapy \\ 15 October 2014 \\ Number of times this article has been viewed
}

\section{Takatsugu Ishimoto Hideo Baba \\ Department of Gastroenterological Surgery, Graduate School of Medical Science, Kumamoto University, Kumamoto, Japan}

\begin{abstract}
Gastric cancer (GC) remains a leading cause of cancer-related deaths worldwide. Despite the recent advance of anticancer drugs and the development of molecular-targeted drugs, the prognosis of patients with advanced GC remains poor, especially in Western countries, and is mainly implicated in tumor relapse and metastasis. Cancer stem cells are selectively capable of tumor initiation and implicated in tumor relapse and metastasis, thus governing the prognosis of GC patients. Recent investigations have indicated that gastric cancer stem cells (GCSCs) are likely to be the most crucial target in GC treatments. Therefore, the identification of key molecules related to GCSCs is expected to contribute toward the extermination of GC. This review presents the current molecular-targeted therapies against GC according to recent clinical trials and the findings regarding GCSCs and their maintenance that will enable the development of novel therapeutic strategies for patients with GC.
\end{abstract}

Keywords: molecular-targeted therapy, cancer stem cells, gastric cancer

\section{Introduction}

Although gastric cancer (GC) rates have been declining for several decades, GC remains the major cause of cancer-related deaths worldwide. ${ }^{1}$ The region of tumors within the stomach has changed over recent years. Tumors of a distal site are less frequent, and tumors of a proximal site are more prevalent. Although complete resection of cancer and extended lymph node dissection are the only curative treatments for GC, the prognosis of patients with advanced $\mathrm{GC}$ after curative resection remains poor, mainly as a result of tumor relapse and metastases. ${ }^{2}$ Therefore, definitive adjunctive chemotherapy for the treatment of GC is urgently needed to improve outcomes.

Increasing evidence has shown that tumor heterogeneity is a result of the hierarchical organization of cancer stem cells (CSCs), which are deeply implicated in tumor relapse and metastasis. ${ }^{3-5}$ Therefore, specific markers to isolate CSCs have been explored. Studies showed that CSCs tended to share cell surface markers with tissue stem cells. CSCs were first reported in acute myeloid leukemia by Bonnet and Dick $^{6}$ in 1997, who found that CD34+CD38- fractions from acute myeloid leukemia patients enhanced tumorigenicity after serial transplantation into immunodeficient mice. CSCs have been subsequently identified in various types of solid tumors. ${ }^{7-9}$ Gastric cancer stem cells (GCSCs) have recently been identified in studies of GC cell lines and primary GC tissues. ${ }^{10}$

This review examines the functional relevance of GCSCs in tumor progression, relapse, and metastasis, and further discusses the potential of molecular-targeted therapy based on targeting GCSCs.
Correspondence: Hideo Baba Department of Gastroenterological Surgery, Graduate School of Medical Science, Kumamoto University,

I-I-I Honjo, Kumamoto 860-8556, Japan

$\mathrm{Tel}+81963735212$

Fax +81963714378

Email hdobaba@kumamoto-u.ac.jp 


\section{Current treatment and outcome of GC patients}

Surgery is currently the only curative modality to treat GC, and the curative rate of surgery alone is $>90 \%$ in stage $1 \mathrm{~A}$ (T1N0M0) GC patients. Endoscopic screening has become widespread, and early GCs are detectable in Eastern Asian countries, such as Korea and Japan, whereas in Western countries, GCs are often detected at an advanced stage and prognosis remains poor. Tumor recurrence is often observed in patients with advanced GC even after complete resection of cancer, indicating that undetectable tumor cells in the bloodstream must be present at the time of surgery. Based on this reason, there is a definitive consensus that multimodality treatments consisting of surgery with neoadjuvant chemotherapy, adjuvant chemotherapy, or chemoradiation would improve outcomes compared with surgery alone. However, the absolute regimen of adjuvant therapy has not been applied globally. Indeed, postoperative adjuvant chemotherapy has been established as the standard of adjunctive treatment in Asia, and postoperative adjuvant chemoradiation therapy is accepted as the standard in North America. Neoadjuvant chemotherapy is taken as a mainstream approach of adjunctive treatment across Europe, the UK, and Australasia.

Recently, several molecular-targeted drugs have been assessed in diverse combinations with conventional chemotherapy versus chemotherapy alone as first-line therapy against advanced GC. The Trastuzumab for Gastric Cancer (ToGA) trial demonstrated that trastuzumab, a recombinant monoclonal antibody against HER2 (also known as ERBB2), combined with fluoropyrimidine plus cisplatin chemotherapy provided a significant survival advantage compared with chemotherapy alone in patients with HER2-positive advanced GC. ${ }^{11}$ The Avastin in Gastric Cancer (AVAGAST) trial evaluated the efficacy of adding bevacizumab, a humanized antihuman vascular endothelial growth factor A (VEGFA) monoclonal antibody, to capecitabine-cisplatin as first-line treatment for advanced GC. The trial demonstrated that the addition of bevacizumab to chemotherapy was associated with significant increases in progression-free survival and overall response rate, but not in overall survival. ${ }^{12}$ The international Erbitux (cetuximab) in combination with Xeloda (capecitabine) and cisplatin in advanced esophagogastric cancer (EXPAND) trial was designed to assess efficacy and safety of addition of cetuximab, a chimeric immunoglobulin G1 monoclonal antibody directed against epidermal growth factor receptor, to capecitabine-cisplatin chemotherapy as a first line in patients with unresectable advanced or metastatic gastric adenocarcinoma. However, this trial demonstrated that addition of cetuximab to capecitabine-cisplatin provided no additional benefit to chemotherapy alone in the first-line treatment of advanced GC. ${ }^{13}$ The REAL-3 trial assessed the addition of panitumumab, a human immunoglobulin G2 monoclonal antibody directed against epidermal growth factor receptor, to epirubicin, oxaliplatin, and capecitabine in patients with advanced esophagogastric adenocarcinoma. Consequently, this trial concluded that addition of panitumumab to capecitabine chemotherapy did not increase overall survival and could not be recommended for use in an unselected population with advanced esophagogastric adenocarcinoma. ${ }^{14}$ The REGARD trial assessed the safety and efficacy of ramucirumab, a monoclonal antibody VEGFR-2 antagonist, in patients with advanced gastric or gastroesophageal junction adenocarcinoma who had disease progression after first-line chemotherapy. Notably, the trial demonstrated that ramucirumab is the first biological treatment given as a single drug that has survival benefits in patients with advanced gastric or gastroesophageal junction adenocarcinoma progressing after first-line chemotherapy. ${ }^{15}$

On the basis of these clinical trials, all unresectable advanced or recurrent GC patients should be screened for HER2 positivity, and patients with HER2-positive GC should receive first-line trastuzumab in combination with platinum plus either fluorouracil or capecitabine. After first-line chemotherapy, ramucirumab could be recommended as one of the options, according to the REGARD trial. ${ }^{15}$

\section{Identification of GC stem cells}

Accumulating evidence has recently shown that CSCs have an enhanced tumor-initiating ability and are deeply implicated in tumor progression and metastasis. Numerous molecules have been examined as candidates for CSC markers to identify the CSC population in various types of cancer. CD44 was first identified as a potential cell surface marker of GCSCs in several GC cell lines. The CD44+ fraction isolated from these cell lines demonstrated a sphere-forming ability in vitro and tumorigenic ability when injected into the stomach wall or skin of immunodeficient mice. ${ }^{16}$ In a previous study, the combination of the cell surface markers CD44 and CD24 was examined in GC cell lines and primary GC tissues from five patients using fluorescence-activated cell sorting. The results demonstrated that the CD44+/CD24+ fraction exhibited higher tumorigenicity when injected into immunodeficient mice compared with the CD44-/CD24-fraction. These cells thus have the capacities to both self-renew and produce differentiated progeny, which suggests that combined $\mathrm{CD} 44+/ \mathrm{CD} 24+$ expression may act as a putative 
GCSC marker. ${ }^{17}$ The combination of CD44 and CD54 surface markers was used to isolate CSCs from human GC tissues and the peripheral blood of GC patients, and the isolated CSCs generated tumors that resembled the original human tumors when injected into immunodeficient mice. The same cells differentiated into gastric epithelial cells in vitro and self-renewed in vivo and in vitro. These results suggest that the combination of CD44+/CD54+ can also be used as a potential biomarker for GCSCs. ${ }^{18}$

The combination of epithelial cell adhesion molecule and CD44 was identified as a putative GCSC marker. The epithelial cell adhesion molecule+/CD44+ fraction from human GC tissues formed tumors in immunodeficient mice and maintained a differentiated phenotype and reproduced the morphological and phenotypical heterogeneities of the original gastric tumor tissues. These cells had greater resistance to anticancer drugs than other cell subpopulations. ${ }^{19}$

A recent study also demonstrated aldehyde dehydrogenase 1 (ALDH1) as a candidate marker for GCSCs. ALDH1+ cells from a human diffuse-type GC cell line possessed a higher tumorigenic capacity in vitro and in vivo compared with ALDH1- cells and were able to self-renew and generate heterogeneous cell populations. Moreover, regenerating islet-derived family member 4 was upregulated in ALDH1+ GCSCs, and ALDH1 and regenerating isletderived family member 4 expression was downregulated by transforming growth factor- $\beta$ (TGF- $\beta$ ), which correlated with a reduction in the GCSC population and tumorigenicity. ${ }^{20}$

A more recent report showed that GCSCs were enriched through spheroid body formation by cultivating the human GC cell line MKN-45 in defined serum-free medium. Spheroid body-forming cells possessed GCSC properties, including persistent self-renewal, extensive proliferation, drug resistance, high tumorigenic capacity, and overexpression of CSC-related genes and proteins (Oct4, Sox2, Nanog, and CD44) compared with the parental cells. ${ }^{21}$

Another study revealed that CD90 may be another potential candidate marker of GCSCs. CD90+ cells possessed a greater ability to initiate tumors in vivo compared with CD90- cells and could re-establish the cellular hierarchy of tumors from single cell implantation, demonstrating their self-renewal properties. Additionally, ERBB2 was overexpressed in approximately $25 \%$ of the gastric primary tumor models, which correlated with the higher level of CD90 expression in these tumors. Trastuzumab treatment could reduce the CD90+ population in the whole tumor mass and suppress tumor growth when combined with traditional chemotherapy. ${ }^{22}$
Unlike expressing markers in CSCs, the CD71- fraction in MKN1 cells was enriched after treatment with 5-fluorouracil and accumulated during the G0/G1 cell-cycle phase. This subpopulation also showed high chemoresistance to conventional chemotherapy, demonstrating its stem cell-like properties. Limiting dilution and serial transplantation assays revealed that the $\mathrm{CD} 71$ - cell fraction had higher tumorigenicity than the CD71+ cell fraction. ${ }^{23}$

Several studies have shown the potential of CD133 as a GCSC marker. The expression of three putative CSC markers, ATP-binding cassette subfamily B member 1, ATP-binding cassette subfamily G member 2, and CD133, were examined in 90 human GC tissue samples and three human GC cell lines. The expression levels of these markers in $\mathrm{GC}$ varied with the degree of differentiation; poorly differentiated GC expressed high levels of these markers. ${ }^{24}$ Another report showed that CD133 expression could be divided into two patterns: luminal expression in the gland and cytoplasmic expression. Multivariate analysis revealed that expression of CD133 in the cytoplasm was an independent prognostic factor in GC. ${ }^{25}$ The GCSC markers reported to date are summarized in Table 1.

\section{Critical analysis of the potential for targeting CSCs in GC}

To identify the potential target for CSCs, we have to single out unique molecules or biological features of CSC. Several molecules have been investigated as target-related specific signaling pathways, cell surface markers, and microenvironmental factors. Several drugs such as salinomycin, metformin, and curcumin have also been identified by chemical screening. Some of these drugs are already tested at early clinical phases of development and will hopefully progress to the stage of clinical application.

We previously used $K 19-W n t 1 / C 2 m E$ mice, a transgenic GC mouse model, to demonstrate that the CD44 variant isoform $(\mathrm{CD} 44 \mathrm{v})$, one of the cell surface markers of GCSC, contributed to defense against reactive oxygen species by stabilizing the glutamate-cystine transporter subunit $\mathrm{xCT}$ and promoting the synthesis of the primary intracellular antioxidant glutathione. ${ }^{26,27}$ Furthermore, CD44v expression was increased in these gastric tumor cells, and inhibition of cystine transport by system xc(-) with sulfasalazine, an inhibitor of xCT-dependent cystine transport, suppressed the development of gastric tumors in these transgenic mice. ${ }^{28}$ Our findings revealed that targeted therapy against the $\mathrm{CD} 44 \mathrm{v}-\mathrm{xCT}$ system may provide a strategy for targeting $\mathrm{CSCs}$ in GC treatment. However, a recent study indicated 
Table I Gastric cancer stem cell markers

\begin{tabular}{|c|c|c|c|c|}
\hline Studies & $\begin{array}{l}\text { Markers } \\
\text { of GCSCs }\end{array}$ & In vitro assay & $\begin{array}{l}\text { In vivo tumorigenicity } \\
\text { (mouse and transplanted site) }\end{array}$ & Source \\
\hline Takaishi et al ${ }^{16}$ & CD44 & $\begin{array}{l}\text { Spheroid formation, } \\
\text { chemoresistance }\end{array}$ & $\begin{array}{l}\text { SCID mice, stomach } \\
\text { and subcutaneous layer }\end{array}$ & $\begin{array}{l}\text { NCI-N87, AGS, MKN-28, } \\
\text { MKN-45, MKN-74 }\end{array}$ \\
\hline Zhang et a $\left.\right|^{17}$ & CD24/CD44 & Spheroid formation & $\begin{array}{l}\text { NOD-SCID mice, } \\
\text { subcutaneous layer }\end{array}$ & AGS, human GC tissues \\
\hline Chen et al ${ }^{18}$ & CD54/CD44 & Spheroid formation & $\begin{array}{l}\text { SCID and nude mice, } \\
\text { subcutaneous layer }\end{array}$ & $\begin{array}{l}\text { Human GC tissues, } \\
\text { peripheral blood }\end{array}$ \\
\hline Han et al ${ }^{19}$ & EрCAM/CD44 & $\begin{array}{l}\text { Spheroid formation, } \\
\text { chemoresistance }\end{array}$ & Nude mice, subcutaneous layer & Human GC tissues \\
\hline Katsuno et $\mathrm{al}^{20}$ & ALDHI & Colony formation & Nude mice, subcutaneous layer & $\begin{array}{l}\text { OCUM-2MLN, HSC-39, HSC- } 43 \text {, } \\
\text { OCUM-I2, OCUM-8, KATO-III, } \\
\text { MKN-7, MKN-74 }\end{array}$ \\
\hline Jiang et $\mathrm{al}^{22}$ & CD90 & Spheroid formation & $\begin{array}{l}\text { Nude mice, subcutaneous layer, } \\
\text { chemoresistance (in vivo) }\end{array}$ & Human GC tissues \\
\hline Ohkuma et $\mathrm{al}^{23}$ & CD7I- & $\begin{array}{l}\text { Colony formation, 3D matrigel } \\
\text { culture, chemoresistance, } \\
\text { tumor cell invasion }\end{array}$ & $\begin{array}{l}\text { NOD-SCID mice, } \\
\text { subcutaneous layer }\end{array}$ & MKN-I \\
\hline Jiang et $\mathrm{al}^{24}$ & CDI33 & Cell proliferation & $\begin{array}{l}\text { Nude mice, stomach and } \\
\text { subcutaneous layer }\end{array}$ & HGC-27, BGC-823, SGC-790I \\
\hline
\end{tabular}

Abbreviations: GCSC, gastric cancer stem cells; SCID, severe combined immunodeficiency; NOD-SCID, nonobese diabetic-severe combined immunodeficiency; GC, gastric cancer; EpCAM, epithelial cell adhesion molecule; ALDHI, aldehyde dehydrogenase I.

that the hierarchical organization involving CSCs and nonCSCs may be reversible through epigenetic gene regulation, ${ }^{29}$ suggesting that therapeutic strategies targeting GCSCs themselves might be insufficient to exterminate cancer cells.

Current evidence suggests that the characteristics of tissue stem cells, including pluripotency and self-renewal, are regulated by the surrounding microenvironment, referred to as the "stem cell niche". Tissue stem cells in the stomach are surrounded by a sheet of myofibroblasts that act as a niche and secrete different types of soluble factors, including bone morphogenetic proteins, TGF- $\beta 1$, Wnt ligands, stromal cellderived factor 1 , and matrix metalloproteinases. ${ }^{30,31} \mathrm{CSCs}$ also depend on a similar niche, called the "CSC niche", which regulates their proliferation and differentiation. ${ }^{32-35}$ The CSC niche is composed of diverse cell lineages, including inflammatory cells, hematopoietic cells, and bone marrowderived myofibroblasts, as well as vasculature, extracellular matrix, and hypoxia. ${ }^{36}$ Several studies showed that targeting the unique molecules in the CSC niche and the signaling interactions between CSCs and the CSC niche may thus be a promising therapeutic strategy and may provide a complementary approach to conventional therapies targeting the malignant cells.

Among the stromal cells, myofibroblasts, also known as carcinoma-associated fibroblasts (CAFs), share characteristics with smooth muscle cells and fibroblasts. CAFs promote the growth of various types of tumors through secretion of soluble factors, including growth factors and cytokines. ${ }^{37,38}$ Recent evidence showed that CAFs significantly increased the number of spheroid colonies and the expression levels of CSC markers in scirrhous GC cell lines, OCUM-12/side population cells, and OCUM-2MD3/side population cells. This effect of CAFs was significantly decreased by TGF- $\beta$ inhibitors but not by fibroblast growth factor receptor or $\mathrm{cMet}$ inhibition. These data suggest that CAFs might regulate CSC properties in scirrhous GC by TGF- $\beta$ signaling. ${ }^{39}$

Hypoxia plays pivotal roles in cell survival, angiogenesis, tumor invasion, and metastasis, and is involved in the maintenance of self-renewal and the undifferentiated state of CSCs in various solid tumors, including glioma, ${ }^{40}$ prostate cancer ${ }^{41}$ colon cancer, ${ }^{42}$ and ovarian cancer. ${ }^{43} \mathrm{~A}$ GC cell line study showed that hypoxia-induced factor- $1 \alpha$ contributed to hypoxia-increased drug resistance of GC cells by suppressing drug-induced apoptosis and upregulating the expression of major drug transporters. ${ }^{44}$ Both acute and chronic hypoxia decreased the radiosensitivity of GC cells by cell-cycle arrest, while reoxygenation enhanced the radiosensitivity of hypoxic cells. ${ }^{45}$ Moreover, recent studies showed that hypoxia stimulated the epithelial-mesenchymal transition in GC cells via autocrine TGF- $\beta /$ TGF- $\beta$ receptor signaling, while intratumoral hypoxia promoted immune tolerance by inducing regulatory T-cells via TGF- $\beta 1{ }^{46,47}$ Taken together, these studies suggest that hypoxia and the related signaling may contribute to the characteristics of GCSCs, although the underlying mechanisms remain unknown. 
Recent evidence demonstrated that CSCs live within a microscopic protective niche formed by blood vessels, called the "vascular niche", which promotes their stemlike and tumorigenic states. Vascular endothelial cells have been identified as a critical component of the CSC niche. Calabrese et al showed that brain CSCs were located nearer to the tumor vasculature than nonstem-like tumor cells in brain tumor xenografts. ${ }^{44}$ Furthermore, they demonstrated that human primary endothelial cells interacted selectively with brain CSCs in culture conditions and secreted factors to support the maintenance and expansion of stem-like tumor cells and to promote their tumorigenicity. Notably, depletion of blood vessels from xenografts ablated self-renewing cells from tumors and arrested tumor growth. ${ }^{48}$ These data indicate that the tumor vasculature may be essential for supporting and preserving the stem-like properties and expansion of CSCs, which are, in turn, critical for their ability to cause tumor progression. As for GCs, a recent study showed that trastuzumab in combination with VEGF-Trap binding to VEGFA, VEGFB, and placental growth factor may represent an effective approach to treating HER2-overexpressing GCs. ${ }^{49}$

Vasculogenic mimicry (VM) has been identified as a new pattern of tumor neovascularization characterized by the acquisition of endothelial cell markers and the formation of vascular channels by tumor cells. VM has been reported in dysregulated melanoma and several other types of malignancies $^{50-54}$ and is associated with an undifferentiated tumor cell phenotype and poor prognosis, suggesting that CSCs may be more likely to participate in this process. ${ }^{55-57}$ The existence of VM was also revealed in GC, especially in poorly differentiated GC, and was related to unfavorable prognosis..$^{58}$ Furthermore, IRX1 overexpression effectively suppressed peritoneal
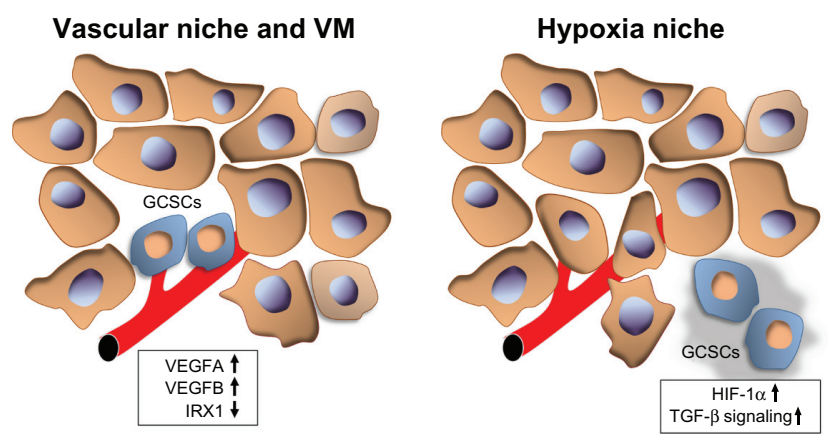

Figure I GCSC niche.

Notes: GCSCs exist as a subpopulation in cancer tissues and their properties are regulated by the GCSC niche. Tumor vasculature (left image) and hypoxia (right image) are likely to play an important role in the GCSC niche through indicated factors. Abbreviations: GCSCs, gastric cancer stem cells; VM, vasculogenic mimicry; VEGFA, vascular endothelial growth factor A; VEGFB, vascular endothelial growth factor B; HIF-I $\alpha$, hypoxia-induced factor-I $\alpha$; TGF- $\beta$, transforming growth factor- $\beta$. spreading and pulmonary metastasis via antiangiogenesis and anti-VM mechanisms, in addition to its previously known effects on cell growth and invasion..$^{59}$ These data indicate that tumor vasculature and hypoxia may play an important role for GCSC properties and suggest that further explorations into the precise mechanisms of VM may lead to new therapeutic strategies aimed at the GCSC niche (Figure 1).

\section{Conclusion}

The treatment strategies for solid tumors have been changed remarkably since the new era of molecular-targeted drugs. These new drugs also have been assessed in diverse combination with conventional chemotherapy as a treatment against advanced GC. However, a successful molecular-targeted drug for GCs has not yet been identified, and the prognosis of patients with advanced GC remains poor. Increasing evidence has recently shown that CSCs tend to be resistant to conventional chemotherapy and are deeply implicated in metastasis and recurrence. Thus, these small populations are recognized as targets of treatment in various types of cancer. Conversely, the CSC niche is known as a regulator of CSC properties, and the identification of definitive molecules in the relationship between CSCs and their niche is required to develop the targeting CSC treatment. This review describes accumulating evidence regarding the unique markers of GCSC and the related molecules with the GCSC niche. Further elucidation of the underlying molecular mechanisms may lead to the development of novel treatment strategies for patients with GC.

\section{Disclosure}

The authors report no conflicts of interest in this work.

\section{References}

1. Bertuccio $P$, Chatenoud L, Levi F, et al. Recent patterns in gastric cancer: a global overview. Int J Cancer. 2009;125(3):666-673.

2. Hohenberger P, Gretschel S. Gastric cancer. Lancet. 2003;362(9380): 305-315.

3. Clarke MF, Fuller M. Stem cells and cancer: two faces of eve. Cell. 2006;124(6):1111-1115.

4. Jordan CT, Guzman ML, Noble M. Cancer stem cells. $N$ Engl J Med. 2006;355(12):1253-1261.

5. Visvader JE, Lindeman GJ. Cancer stem cells: current status and evolving complexities. Cell Stem Cell. 2012;10(6):717-728.

6. Bonnet D, Dick JE. Human acute myeloid leukemia is organized as a hierarchy that originates from a primitive hematopoietic cell. Nat Med. 1997;3(7):730-737.

7. Al-Hajj M, Wicha MS, Benito-Hernandez A, Morrison SJ, Clarke MF. Prospective identification of tumorigenic breast cancer cells. Proc Natl Acad Sci U S A. 2003;100(7):3983-3988.

8. Singh SK, Clarke ID, Terasaki M, et al. Identification of a cancer stem cell in human brain tumors. Cancer Res. 2003;63(18):5821-5828.

9. Ricci-Vitiani L, Lombardi DG, Pilozzi E, et al. Identification and expansion of human colon-cancer-initiating cells. Nature. 2007;445(7123): $111-115$. 
10. Xu G, Shen J, Ou Yang X, Sasahara M, Su X. Cancer stem cells: the 'heartbeat' of gastric cancer. J Gastroenterol. 2013;48(7):781-797.

11. Bang YJ, Van Cutsem E, Feyereislova A, et al. Trastuzumab in combination with chemotherapy versus chemotherapy alone for treatment of HER2-positive advanced gastric or gastro-oesophageal junction cancer (ToGA): a phase 3, open-label, randomised controlled trial. Lancet. 2010;376(9742):687-697.

12. Ohtsu A, Shah MA, Van Cutsem E, et al. Bevacizumab in combination with chemotherapy as first-line therapy in advanced gastric cancer: a randomized, double-blind, placebo-controlled phase III study. J Clin Oncol. 2011;29(30):3968-3976.

13. Lordick F, Kang YK, Chung HC, et al. Capecitabine and cisplatin with or without cetuximab for patients with previously untreated advanced gastric cancer (EXPAND): a randomised, open-label phase 3 trial. Lancet Oncol. 2013;14(6):490-499.

14. Waddell T, Chau I, Cunningham D, et al. Epirubicin, oxaliplatin, and capecitabine with or without panitumumab for patients with previously untreated advanced oesophagogastric cancer (REAL3): a randomised, open-label phase 3 trial. Lancet Oncol. 2013;14(6):481-489.

15. Fuchs CS, Tomasek J, Yong CJ, et al. Ramucirumab monotherapy for previously treated advanced gastric or gastro-oesophageal junction adenocarcinoma (REGARD): an international, randomised, multicentre, placebo-controlled, phase 3 trial. Lancet. 2014;383(9911):31-39.

16. Takaishi S, Okumura $\mathrm{T}$, Tu S, et al. Identification of gastric cancer stem cells using the cell surface marker CD44. Stem Cells. 2009;27(5): 1006-1020.

17. Zhang C, Li C, He F, Cai Y, Yang H. Identification of CD44+CD24+ gastric cancer stem cells. J Cancer Res Clin Oncol. 2011;137(11): $1679-1686$.

18. Chen T, Yang K, Yu J, et al. Identification and expansion of cancer stem cells in tumor tissues and peripheral blood derived from gastric adenocarcinoma patients. Cell Res. 2012;22(1):248-258.

19. Han ME, Jeon TY, Hwang SH, et al. Cancer spheres from gastric cancer patients provide an ideal model system for cancer stem cell research. Cell Mol Life Sci. 2011;68(21):3589-3605.

20. Katsuno Y, Ehata S, Yashiro M, Yanagihara K, Hirakawa K, Miyazono K. Coordinated expression of REG4 and aldehyde dehydrogenase 1 regulating tumourigenic capacity of diffuse-type gastric carcinoma-initiating cells is inhibited by TGF-beta. $J$ Pathol. 2012;228(3):391-404.

21. Liu J, Ma L, Xu J, et al. Spheroid body-forming cells in the human gastric cancer cell line MKN-45 possess cancer stem cell properties. Int J Oncol. 2013;42(2):453-459.

22. Jiang J, Zhang Y, Chuai S, et al. Trastuzumab (herceptin) targets gastric cancer stem cells characterized by CD90 phenotype. Oncogene. 2012;31(6):671-682.

23. Ohkuma M, Haraguchi N, Ishii $\mathrm{H}$, et al. Absence of CD71 transferrin receptor characterizes human gastric adenosquamous carcinoma stem cells. Ann Surg Oncol. 2012;19(4):1357-1364.

24. Jiang $\mathrm{Y}, \mathrm{He} \mathrm{Y}, \mathrm{Li} \mathrm{H}$, et al. Expressions of putative cancer stem cell markers $\mathrm{ABCB} 1, \mathrm{ABCG}$, and $\mathrm{CD} 133$ are correlated with the degree of differentiation of gastric cancer. Gastric Cancer. 2012;15(4): $440-450$.

25. Hashimoto K, Aoyagi K, Isobe T, Kouhuji K, Shirouzu K. Expression of CD133 in the cytoplasm is associated with cancer progression and poor prognosis in gastric cancer. Gastric Cancer. 2014;17(1): 97-106.

26. Ishimoto $T$, Nagano $O$, Yae $T$, et al. CD44 variant regulates redox status in cancer cells by stabilizing the $\mathrm{xCT}$ subunit of system xc(-) and thereby promotes tumor growth. Cancer Cell. 2011;19(3):387-400.

27. Nagano O, Okazaki S, Saya H. Redox regulation in stem-like cancer cells by CD44 variant isoforms. Oncogene. 2013;32(44):5191-5198.

28. Wada T, Ishimoto T, Seishima R, et al. Functional role of CD44v-xCT system in the development of spasmolytic polypeptide-expressing metaplasia. Cancer science. 2013;104(10):1323-1329.

29. Roesch A, Fukunaga-Kalabis M, Schmidt EC, et al. A temporarily distinct subpopulation of slow-cycling melanoma cells is required for continuous tumor growth. Cell. 2010;141(4):583-594.
30. Leedham SJ, Brittan M, Preston SL, McDonald SA, Wright NA. The stomach periglandular fibroblast sheath: all present and correct. Gut. 2006;55(2):295-296.

31. Hoffmann W. Stem cells, self-renewal and cancer of the gastric epithelium. Curr Med Chem. 2012;19(35):5975-5983.

32. Li L, Neaves WB. Normal stem cells and cancer stem cells: the niche matters. Cancer Res. 2006;66(9):4553-4557.

33. Visvader JE, Lindeman GJ. Cancer stem cells in solid tumours: accumulating evidence and unresolved questions. Nat Rev Cancer. 2008;8(10):755-768.

34. Boral D, Nie D. Cancer stem cells and niche mircoenvironments. Front Biosci (Elite Ed). 2012;4:2502-2514.

35. Yi SY, Hao YB, Nan KJ, Fan TL. Cancer stem cells niche: a target for novel cancer therapeutics. Cancer Treat Rev. 2013;39(3):290-296.

36. Borovski T, De Sousa EMF, Vermeulen L, Medema JP. Cancer stem cell niche: the place to be. Cancer Res. 2011;71(3):634-639.

37. Orimo A, Gupta PB, Sgroi DC, et al. Stromal fibroblasts present in invasive human breast carcinomas promote tumor growth and angiogenesis through elevated SDF-1/CXCL12 secretion. Cell. 2005;121(3):335-348.

38. Joyce JA, Pollard JW. Microenvironmental regulation of metastasis. Nat Rev Cancer. 2009;9(4):239-252.

39. Hasegawa T, Yashiro M, Nishii T, et al. Cancer-associated fibroblasts might sustain the stemness of scirrhous gastric cancer cells via transforming growth factor-beta signaling. Int $J$ Cancer. 2014;134(8): $1785-1795$.

40. Soeda A, Park M, Lee D, et al. Hypoxia promotes expansion of the CD133-positive glioma stem cells through activation of HIF-1alpha. Oncogene. 2009;28(45):3949-3959.

41. Ma Y, Liang D, Liu J, et al. Prostate cancer cell lines under hypoxia exhibit greater stem-like properties. PLoS One. 2011;6(12):e29170.

42. Yeung TM, Gandhi SC, Bodmer WF. Hypoxia and lineage specification of cell line-derived colorectal cancer stem cells. Proc Natl Acad Sci U S A. 2011;108(11):4382-4387.

43. Liang D, MaY, Liu J, et al. The hypoxic microenvironment upgrades stemlike properties of ovarian cancer cells. BMC Cancer. 2012;12:201.

44. Liu L, Ning X, Sun L, et al. Hypoxia-inducible factor-1 alpha contributes to hypoxia-induced chemoresistance in gastric cancer. Cancer Sci. 2008;99(1):121-128.

45. Kato Y, Yashiro M, Fuyuhiro Y, et al. Effects of acute and chronic hypoxia on the radiosensitivity of gastric and esophageal cancer cells. Anticancer Res. 2011;31(10):3369-3375.

46. Matsuoka J, Yashiro M, Doi Y, et al. Hypoxia stimulates the EMT of gastric cancer cells through autocrine TGFbeta signaling. PLoS One. 2013;8(5):e62310.

47. Deng B, Zhu JM, Wang Y, et al. Intratumor hypoxia promotes immune tolerance by inducing regulatory $\mathrm{T}$ cells via TGF-beta1 in gastric cancer. PLoS One. 2013;8(5):e63777.

48. Calabrese C, Poppleton H, Kocak M, et al. A perivascular niche for brain tumor stem cells. Cancer Cell. 2007;11(1):69-82.

49. Singh R, Kim WJ, Kim PH, Hong HJ. Combined blockade of HER2 and VEGF exerts greater growth inhibition of HER2-overexpressing gastric cancer xenografts than individual blockade. Exp Mol Med. 2013;45:e52.

50. Maniotis AJ, Folberg R, Hess A, et al. Vascular channel formation by human melanoma cells in vivo and in vitro: vasculogenic mimicry. Am J Pathol. 1999;155(3):739-752.

51. Shirakawa K, Kobayashi H, Heike Y, et al. Hemodynamics in vasculogenic mimicry and angiogenesis of inflammatory breast cancer xenograft. Cancer Res. 2002;62(2):560-566.

52. Millimaggi D, Mari M, D’Ascenzo S, Giusti I, Pavan A, Dolo V. Vasculogenic mimicry of human ovarian cancer cells: role of CD147. Int J Oncol. 2009;35(6):1423-1428.

53. Liu Z, Sun B, Qi L, Li H, Gao J, Leng X. Zinc finger E-box binding homeobox 1 promotes vasculogenic mimicry in colorectal cancer through induction of epithelial-to-mesenchymal transition. Cancer Sci. 2012;103(4):813-820. 
54. Wu S, Yu L, Wang D, et al. Aberrant expression of CD133 in non-small cell lung cancer and its relationship to vasculogenic mimicry. $B M C$ Cancer. 2012;12:535.

55. Yao XH, Ping YF, Bian XW. Contribution of cancer stem cells to tumor vasculogenic mimicry. Protein Cell. 2011;2(4):266-272.

56. Liu TJ, Sun BC, Zhao XL, et al. CD133+ cells with cancer stem cell characteristics associates with vasculogenic mimicry in triple-negative breast cancer. Oncogene. 2013;32(5):544-553.
57. Cao Z, Bao M, Miele L, Sarkar FH, Wang Z, Zhou Q. Tumour vasculogenic mimicry is associated with poor prognosis of human cancer patients: a systemic review and meta-analysis. Eur J Cancer. 2013;49(18):3914-3923.

58. Li M, GuY, Zhang Z, et al. Vasculogenic mimicry: a new prognostic sign of gastric adenocarcinoma. Pathol Oncol Res. 2010;16(2):259-266.

59. Jiang J, Liu W, Guo X, et al. IRX1 influences peritoneal spreading and metastasis via inhibiting BDKRB2-dependent neovascularization on gastric cancer. Oncogene. 2011;30(44):4498-4508.

\section{Publish your work in this journal}

Gastrointestinal Cancer: Targets and Therapy is an international, peer-reviewed, open access journal focusing on gastro-intestinal cancer research, identification of therapeutic targets and the optima use of preventative and integrated treatment interventions to achieve improved outcomes, enhanced survival and quality of life for the cancer patient. The manuscript management system is completely online and includes a very quick and fair peer-review system. Visit http://www.dovepress.com/testimonials.php to read real quotes from published authors.

Submit your manuscript here: http://www.dovepress.com/gastro-intestinal-cancer-targets-and-therapy-journal 